\title{
A Specific Brain Tract Guides Follower Growth Cones in Two Regions of the Zebrafish Brain
}

\author{
Ajay B. Chitnis, ${ }^{1, *}$ Chetan K. Patel, ${ }^{2}$ Stennis Kim, ${ }^{2}$ and John Y. Kuwada ${ }^{1,2, \dagger}$ \\ ${ }^{1}$ Neuroscience Program and ${ }^{2}$ Department of Biology, University of Michigan, Ann Arbor, \\ Michigan 48109-1048
}

\section{SUMMARY}

Neurons of the nucleus of the posterior commissure (nuc PC), an identifiable cluster of neurons in the embryonic zebrafish brain, project growth cones ventrally along the posterior commissure to the anterior tegmentum where the PC intersects two longitudinal tracts, the tract of the postoptic commissure (TPOC) and the medial longitudinal fasciculus (MLF). Once at the intersection, nuc PC growth cones turn posteriorly onto the TPOC in the dorsal tegmentum and follow it to the hindbrain. Previously we showed that in the absence of the TPOC, nuc PC growth cones often extended along aberrant pathways suggesting that fasciculation, that is, contact with TPOC axons is an important factor in guiding growth cones along their normal pathway. However, a significant number of nuc PC growth cones also followed their normal pathway suggesting that cues associated with the dorsolateral tegmentum, independent of the TPOC, can also guide nuc PC growth cones. We have now confirmed using electron microscopy that nuc $\mathrm{PC}$ growth cones fasciculate with axons in the TPOC. In the absence of the TPOC, the nuc PC growth cones that extend along their normal pathway do so in contact with dorsolateral neuroepithelial cells. This suggests that cues associated with these cells can also guide the nuc PC growth cones. Furthermore, in the absence of the TPOC axons, these growth cones now inappropriately turn onto axons that normally intersect the TPOC near the border of the midbrain and hindbrain, that is, at a second intersection of tracts. This suggests that fasciculation with TPOC axons may also guide nuc PC growth cones in this second region of the brain. (C) 1992 John Wiley \& Sons, Inc.

Keywords: axonal guidance, growth cone, zebrafish, nucleus of the posterior commissure, axonogenesis.

\section{INTRODUCTION}

Generally, growth cones find their targets by following precise, cell-specific pathways. They accomplish this in a variety of species by interacting with a number of different elements in their environment (Goodman et al., 1984; Landmesser, 1986; Dodd and Jessell, 1988). Recent investigations demonstrated that this is likely the case in the brain of early zebrafish embryos as well (Chitnis and $\mathrm{Ku}$ wada, 1991). These experiments took advantage of

Received April 1, 1992; accepted May 18, 1992.

Journal of Neurobiology, Vol. 23, No. 7, pp. 845-854 (1992)

(C) 1992 John Wiley \& Sons, Inc.

CCC 0022-3034/92/070845-10

* Present address: Salk Institute, P.O. Box 85800, San Diego, CA $92186-5800$.

To whom correspondence should be addressed. the simplicity of the early zebrafish brain: it contains a simple scaffold of axon tracts (Chitnis and Kuwada, 1990; Wilson, Ross, Parrett, and Easter, 1990 ). In the midbrain, the scaffold consists of a bilateral pair of longitudinal tracts, the tract of the postoptic commissure (TPOC) and the medial longitudinal fasciculus (MLF), connected by the posterior commissure (PC) (Fig. 1). The TPOC originates from neurons in the forebrain and extends through the dorsolateral tegmentum to the hindbrain where it becomes continous with the dorsal longitudinal fasciculus (DLF). The MLF, which is ventromedial to the TPOC, originates from neurons in the floor of the anterior midbrain and extends caudally to the spinal cord. The PC is a dorsal commissure located at the junction of the forebrain and midbrain.

The nucleus of the posterior commissure (nuc 
$\mathrm{PC}$ ) is located in the dorsolateral brain just posterior to the PC. Neurons in the nuc PC project growth cones that extend along a stereotyped trajectory within the early brain scaffold (Chitnis and Kuwada, 1990). They first extend ventrally along the PC to an intersection in the anterior tegmentum where the PC intersects the two longitudinal tracts. At this intersection they turn to extend caudally along the TPOC axons to the hindbrain. When the TPOC was surgically prevented from entering the midbrain, nuc PC growth cones extended to the intersection but then often followed aberrant pathways (Chitnis and Kuwada, 1991). However, a significant number of nuc PC growth cones also followed an apparently normal longitudinal pathway in the dorsolateral tegmentum despite the absence of the TPOC. These findings suggested that fasciculation with the TPOC axons normally participates in guiding nuc PC growth cones at this intersection, but that other cues in the dorsolateral tegmentum may also be capable of guiding nuc $\mathrm{PC}$ growth cones.

We have used electron microscopy (EM) to determine the substrates for nuc PC growth cones. (1) Do the nuc PC growth cones normally fasciculate, that is, extend in contact with TPOC axons? (2) When nuc PC growth cones follow an apparently normal pathway in the absence of the TPOC, what do they extend upon in the dorsolateral tegmentum? We also tested the role the TPOC plays in guiding the nuc $\mathrm{PC}$ growth cones beyond the tegmental intersection by examining those growth cones that followed their normal pathway through the midbrain despite the elimination of the TPOC. Our findings confirm that nuc PC growth cones normally fasciculate with TPOC axons and identify the endfeet of neuroepithelial cells in the dorsolateral tegmentum as a possible source of additional guidance cues. Furthermore, we find that the TPOC participates in guiding nuc PC growth cones at a second intersection of tracts located near the midbrain/hindbrain border.

\section{METHODS}

\section{Animals}

Zebrafish embryos were collected from a laboratory breeding colony and maintained according to the procedures described in Myers, Eisen, and Westerfield (1986). They were maintained at $28.5^{\circ} \mathrm{C}$ and staged according to time postfertilization (PF).

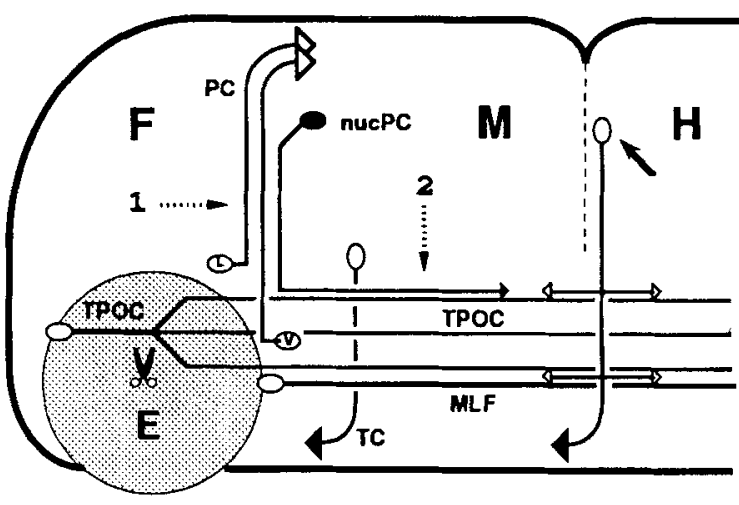

Figure 1 Schematic of a 28-h-old embryo showing a part of the early axon scaffold in a side view of the forebrain $(F)$, midbrain $(M)$, and hindbrain $(H)$. The anterior tegmental intersection of tracts is near the forebrain / midbrain border and the anterior hindbrain intersection is near the midbrain/hindbrain border (dashed line). Open circles represent clusters of neurons that establish the axon scaffold; $\mathrm{L}$ and $\mathrm{V}$ denote, respectively, the lateral and ventral clusters of posterior commissure neurons that pioneer the $\mathrm{PC}$. An arrow indicates the cluster of cells found in the anterior hindbrain that intersect the longitudinal tracts. TC, tegmental commissure; E, eye; other abbreviations as per text. The pair of scissors marks the approximate site of the cut made to prevent the TPOC axons from extending beyond the diencephalon (see text). Arrows labeled 1 and 2 indicate the relative positions and planes of section of the electron micrographs shown in Figure 2(A) and (B), respectively. In this and all other figures, unless specified, anterior is to the left and dorsal is up. For clarity, tracts in the forebrain other than the TPOC are not shown.

\section{Labeling and Electron Microscopic Examination of Growth Cones}

Normal or manipulated embryos at 26-28 h PF were dechorionated, dissected to squeeze out some yolk from their yolk sacs, pinned on their side in a Sylgard-lined embryo holder, and fixed for $4-12 \mathrm{~h}$ in $4 \%$ paraformaldehyde and $0.1 \%$ glutaraldehyde in $0.1 \mathrm{M}$ phosphate buffer ( $\mathrm{pH} 7.4$ ). They were then washed, and placed on a fixed-stage Zeiss compound microscope outfitted with differential interference contrast (DIC) and epifluorescence optics. Nuc PC growth cones were labeled orthogradely by pressure injection of small amounts of a $0.25 \%$ solution of $1,1^{\prime}$-dioctadecyl-3,3,3', $3^{\prime}$-tetramethylindocarbocyanine perchlorate (dil), in $N, N$-dimethylformamide (Honig and Hume, 1986) from a microelectrode into the nuc PC. This cluster can be identified with DIC optics as a group of three to four cells with relatively large cell bodies lateral to the dorsal midline and posterior to the PC. The PC at $28 \mathrm{~h}$ can be recognized as a refractile band at the junction of the forebrain and midbrain. Dil was allowed to spread for $4-12 \mathrm{~h}$, and the 
labeled neurons were marked with a brown reaction product by following the photooxidation procedure with diaminobenzidine (DAB) and epifluorescent illumination (Maranto, 1982). The brown photooxidation product is electron dense and can be clearly distinguished in electron micrographs. After photooxidation the trajectories taken by the labeled nuc PC growth cones were recorded, and the embryos were processed for EM ( $\mathrm{Ku}$ wada, Bernhardt, and Chitnis, 1990). Coronal or horizontal sections were made to examine the growth cones as they were extending ventrally along the $\mathrm{PC}$ to the intersection and at the anterior tegmental intersection. Coronal sections were made to examine growth cones as they extended longitudinally through the tegmentum.

\section{Double Labeling of nuc PC Growth Cones and the Axon Scaffold}

Embryos were prepared as described above for dil labeling but no glutaraldehyde was used in the fixative. Nuc PC growth cones were labeled with dil and photooxidized with $\mathrm{DAB}$. Axons were then labeled with the acetylated $\alpha$-tubulin antibody (Piperno and Fuller, 1985) using the whole-mount labeling procedure previously described (Chitnis and Kuwada, 1991). In this procedure anti-tubulin-labeled axons were violet/blue, whereas the dil-labeled growth cones and axons were brown.

\section{Elimination of the TPOC}

The TPOC axons were prevented from growing into the midbrain by stabilizing embryos ( $16-17 \mathrm{~h}$ or $19-20 \mathrm{~h}$ ) in a drop of $2 \%-3 \%$ methyl cellulose in embryo-rearing solution (Kirchen and West, 1976) and cutting the posterior portion of the forebrain with a pair of fine scissors. Care was taken not to damage the yolk sack during the cut. Embryos were incubated in sterile, Earles Balanced Salt Solution with an antibiotic/antimycotic cocktail (Sigma) or Hank's solution to 27-28 h PF.

\section{Analyses of Growth Cone Behavior at the Midbrain/Hindbrain Border}

The behavior of the nuc PC growth cones at the anterior hindbrain intersection of tracts was analyzed by comparing camera lucida drawings of nuc PC growth cones from 30 normal embryos with those from 13 embryos in which the TPOC had been prevented from entering the midbrain. The number of nuc PC growth cones that were oriented within $45^{\circ}$ to the vertical axis was compared in the two sets of embryos. The vertical axis was defined as the line denoting the plane of the boundary between the midbrain and hindbrain. This plane could be visualized with Nomarski optics in the whole mounts and was drawn as a reference for each embryo examined using camera lucida. A line was drawn between the distal tip (not including filopodia) and base of the growth cone to define the axis of the growth cone. The angle between the vertical axis and the axis of the growth cone was measured. The growth cones were put in two categories: (1) dorsal: those whose axis made an angle $\leq 45^{\circ}$ to the vertical axis; and (2) longitudinal: those whose axis was at an angle $>45^{\circ}$ to the vertical.

\section{RESULTS}

\section{Nuc PC Growth Cones Fasciculate First with PC Axons and then with TPOC Axons}

Nuc PC growth cones follow a specific pathway within the early scaffold of brain tracts by first extending ventrally along the PC, then turning onto the TPOC to extend posteriorly through the dorsolateral tegmentum to the hindbrain (Fig. 1) (Chitnis and Kuwada, 1990). In order to see if nuc PC growth cones do this by fasciculating with PC axons then with TPOC axons, we examined them with electron microscopy at various points along their pathway in normal embryos. The growth cones were marked with a DAB reaction product by labeling them with dil followed by photooxidation. As nuc PC growth cones $(n=3$, from two embryos) extended ventrally along the PC toward the TPOC, they fasciculated with axons located in the region where the PC is normally found and, therefore, were presumed to be PC axons [Fig. $2(A)]$. These axons were recognized in horizontal sections as a cluster of small round profiles, some of which contained microtubules. All the growth cones made extensive contacts with the axons and also contacted other unidentified processes which are likely to be the endfeet of neuroepithelial cells, other growth cones, or glia processes. Because the labeled nuc PC growth cone may have been preceded by other nuc PC axons, the labeled growth cones could contact earlier nuc PC axons and/or the axons of the lateral and ventral PC cluster of neurons that establish the PC. Because labeled growth cones contacted nearly all the axonal profiles, it is likely that they extend ventrally, at least partly, in contact with the axons of the lateral and ventral PC clusters.

Once in the tegmentum nuc $\mathrm{PC}$ growth cones ( $n$ $=3$, from two embryos) extended posteriorly by fasciculating with axons in the TPOC [Fig. 2(B)]. The TPOC breaks up into several parallel bundles prior to the tegmentum (Chitnis and Kuwada, 1990). As before (Chitnis and Kuwada, 1991), these bundles could be easily distinguished in their expected location in the dorsolateral tegmentum 

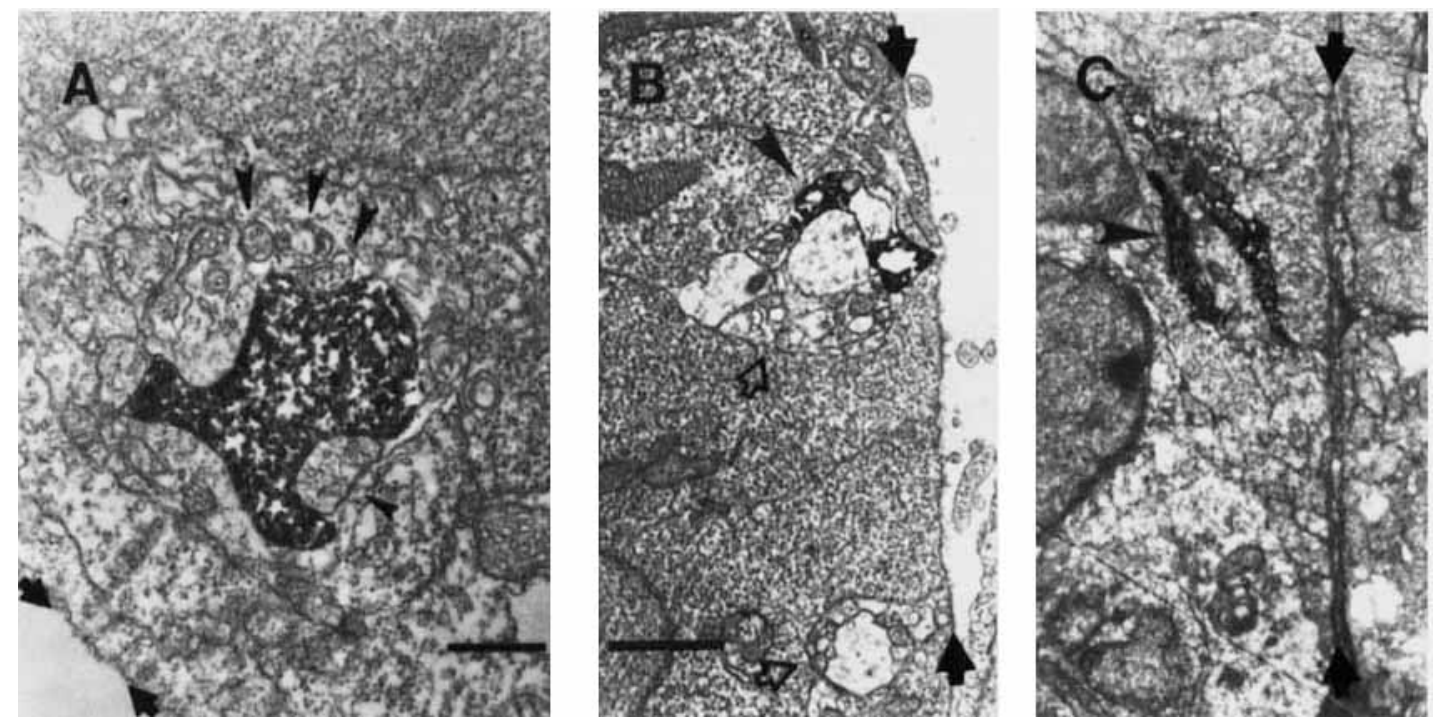

Figure 2 nuc PC growth cones normally fasciculate with pioneer axons, but in the absence of the pioneer axons they extend on the endfeet of neuroepithelial cells. Electron micrographs of photooxidized, dil-labeled nuc PC growth cones. (A) Horizontal section showing a nuc PC growth cone extending ventrally in contact with unlabeled axons (arrowheads) in the PC and unidentified oblong profiles ( see text). The location and orientation of the section is indicated by arrow 1 in Figure 1. The superficial basal lamina is denoted by the arrows in all sections. (B) Coronal section showing parts of a nuc PC growth cone (arrowhead) extending posteriorly in contact with unlabeled TPOC axons in a normal embryo. Two TPOC bundles (open arrows) can be seen. The location and orientation of the section is indicated by arrow 2 in Figure 1. (C) A coronal section showing parts of a nuc $\mathrm{PC}$ growth cone in a region corresponding to that seen in (B) in an embryo in which the TPOC axons were prevented from growing into the midbrain. The growth cone is in contact with processes that are likely to be the endfeet of neuroepithelial cells. Dorsal is up in (B) and (C). Scale bar $=1 \mu \mathrm{m}$ for (A); $5 \mu \mathrm{m}$ for (B) and (C).

55-75 $\mu \mathrm{m}$ away from the ventral midline. The other longitudinal tract in the tegmentum, the MLF, could be seen in these sections as a large, tightly fasciculated tract of axons approximately 35 $\mu \mathrm{m}$ from the midline (not shown). All three nuc PC growth cones fasciculated with the most dorsal TPOC bundle, but examination of other nuc PC growth cones at the light level showed that this was not always the case (see below). Electron microscopic examination of nuc PC growth cones demonstrated that they made extensive contacts with axons in the TPOC bundles and surrounded endfeet of neuroepithelial cells, but rarely contacted the basal lamina. Similarly, the pioneering growth cones of epiphysial neurons in the zebrafish forebrain (Wilson and Easter, 1991) and RohonBeard neurons in the spinal cord of the Japanese Medaka fish (Kuwada, 1986) extend upon the endfeet of neuroepithelial cells with little contact of the basal lamina. Axons in the TPOC include both nuc $P C$ axons and the axons of the diencephalic TPOC neurons that establish the TPOC. Because the majority of axons in the TPOC in the midbrain belong to the diencephalic neurons, it seems likely that nuc PC growth cones extend longitudinally in contact with some TPOC axons.

The turning behavior of nuc PC growth cones at the intersection in the anterior tegmentum was examined in more detail by labeling the growth cones $(n=23)$ with dil followed by photooxidation and labeling all axon tracts including the TPOC bundles and the MLF with an antibody against acetylated $\alpha$-tubulin in 13 embryos. These procedures marked the nuc PC growth cone axons brown and the other axons violet/blue. As shown previously, nuc PC growth cones extend along the PC toward the tegmental intersection $(n=4)$. At the intersection the growth cones switch from the PC $(n=5)$ to a TPOC bundle $(n=7)$, which they followed posteriorly $(n=7)$. The nuc PC growth cones and associated filopodia did not contact the MLF nor did they apparently show any preference for a particular TPOC bundle since they followed any of the bundles (Fig. 3). Occasionally, two nuc PC growth 


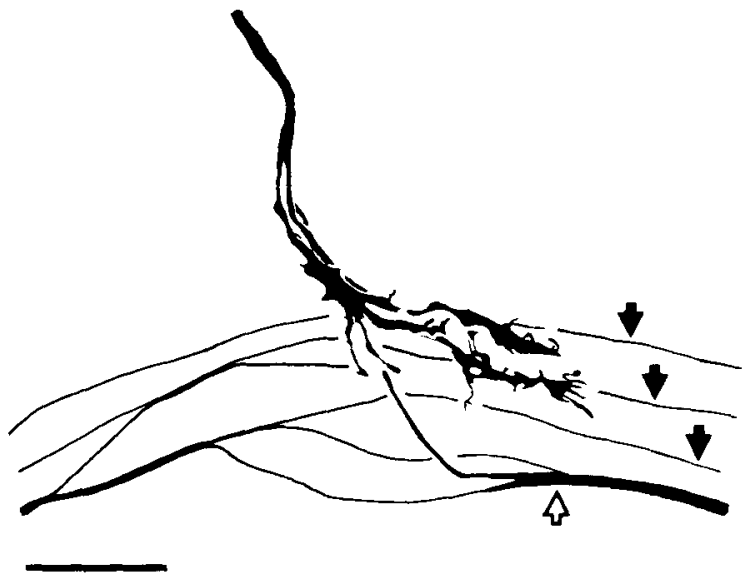

Figure 3 nuc PC growth cones need not follow each other. A camera lucida drawing showing three dil-labeled nuc PC growth cones turning onto TPOC bundles at the anterior tegmental intersection seen in a sideview. The PC, TPOC bundles, and MLF were labeled with a monoclonal antibody (MAb) against acetylated $\alpha$-tubulin. Closed arrows indicate TPOC axon bundles and the open arrow indicates the MLF. Some axons switch from the TPOC to the MLF as they extend posteriorly. Two nuc PC growth cones extend on two separate TPOC bundles. None of the growth cones contacted the MLF. Scale bar $=25 \mu \mathrm{m}$.

cones were observed extending on two separate TPOC bundles.

\section{In the Absence of the TPOC, nuc PC Growth Cones Can Extend on Neuroepithelial Cells}

Some nuc PC growth cones had been previously shown to follow an apparently normal pathway through the dorsolateral tegmentum despite the absence of the TPOC (Chitnis and Kuwada, 1991). We examined such nuc $P C$ growth cones from three embryos with electron microscopy to confirm the removal of the TPOC axons and to identify upon what the growth cones were extending [ Fig. 2(C)]. In all three embryos examined, the TPOC bundles were absent from the region of the dorsolateral tegmentum where the bundles are normally located (between 55 and $75 \mu \mathrm{m}$ lateral to the ventral midline). The labeled growth cones extended longitudinally in contact with the soma of spindle-shaped cells that contact the lumenal basal lamina and numerous profiles found near the superficial basal lamina. The spindle-shaped cells are likely to be neuroepithelial cells from the dorsolateral tegmentum, and the profiles may be their end- feet. As in normal embryos, the growth cones rarely contacted the basal lamina.

\section{Nuc PC Growth Cones Turn Predominantly in a Posterior Direction in the Anterior Tegmentum in the Absence of the TPOC}

The elimination of the TPOC from the anterior tegmental intersection induced many nuc PC growth cones to turn onto inappropriate pathways after reaching the anterior tegmentum (Chitnis and Kuwada, 1991). Of the 23 growth cones that followed aberrant pathways, 13 turned posteriorly and followed the MLF; three turned posteriorly and followed inappropriate pathways independent of other axons; five turned anteriorly and extended to the dorsoventral diencephalic tract (DVDT), a tract that normally intersects the TPOC in the diencephalon, and then extended dorsally along the DVDT; and two made a $180^{\circ}$ turn and extended back dorsally along the PC. Of these axons, $70 \%$ turned posteriorly in the intersection. Overall, including the 36 growth cones that followed an apparently normal pathway despite the elimination of the TPOC, $88 \%$ turned posteriorly. This suggests that directionality cues independent of the TPOC are available to direct nuc $\mathrm{PC}$ growth cones in the intersection.

\section{Nuc PC Growth Cones Occasionally Turn in a Dorsal Direction at an Intersection of Tracts Near the Midbrain/Hindbrain Boundary}

Nuc PC growth cones normally extended along the TPOC axons into the hindbrain between 24 and 28 $\mathrm{h}$ PF [Fig. 4(A), 5(C), 6(A)]. Just posterior to the boundary between the midbrain and hindbrain, the nuc PC growth cones occasionally turned dorsal. Apparently this turn was transient or these axons were later eliminated since at $31 \mathrm{~h}$ PF none of 16 nuc $\mathrm{PC}$ axons from seven normal embryos extended dorsally at this site [Fig. 4(B)]. At this site the acetylated $\alpha$-tubulin antibody labeled ventrally extending axons that intersected the TPOC and MLF $[n=31$; Fig. $5(\mathrm{~A})]$. The earliest of these axons were projected from a group of neurons in the rostral end of the hindbrain and intersected the longitudinal tracts by $18-20 \mathrm{~h} \mathrm{PF}$. These axons of the anterior hindbrain neurons eventually extended both anteriorly and posteriorly along the TPOC and MLF as well as across the ventral midline (not shown). Therefore, this anterior hind- 


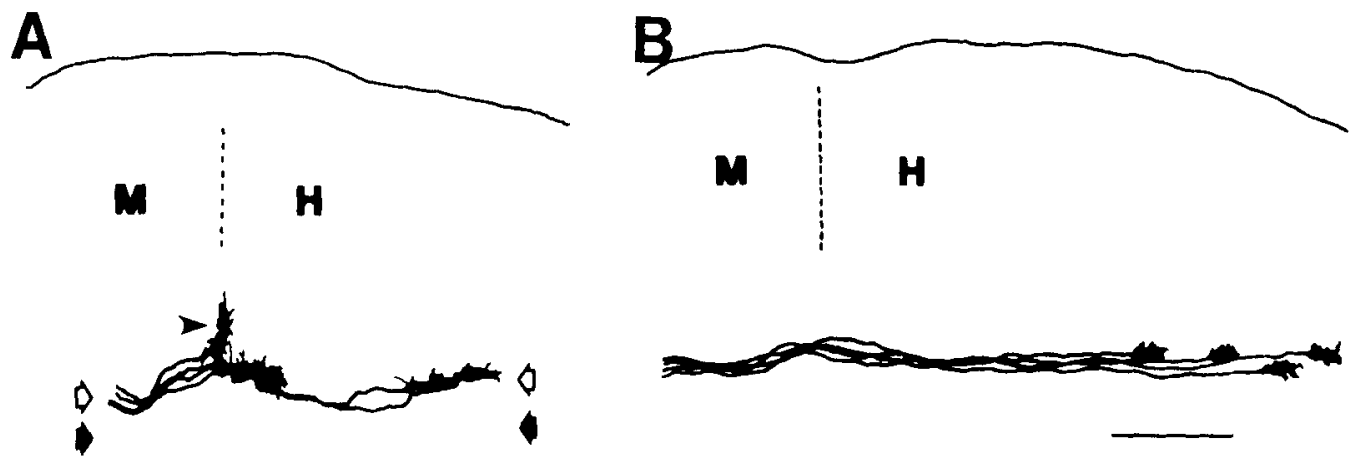

Figure 4 nuc PC growth cones occasionally and transiently turn dorsal near the midbrain (M)/hindbrain ( $\mathrm{H}$ ) border. Camera lucida drawings of dil-labeled nuc PC growth cones. (A) One of the four labeled growth cones ( small arrowheads) turned dorsally at $27 \mathrm{~h} \mathrm{PF}$. Open and closed arrowheads denote the location of the TPOC and MLF, respectively. (B) All four labeled growth cones in another embryo extend longitudinally with little deviation past the midbrain/hindbrain border at $31 \mathrm{~h}$ PF. Scale bar $=50 \mu \mathrm{m}$.

brain intersection was present from 6 to $10 \mathrm{~h}$ prior to the time nuc PC growth cones reached this site and could, in principle, be the substrate for the occasional growth cone that turns dorsally at this site. Concordantly, labeling nuc PC growth cones with dil and other axons with the acetylated $\alpha$-tubulin antibody ( $n=22$ ) showed that the nuc PC growth cones that extend dorsally do so by following these hindbrain axons [Fig. 5(C)].

\section{More Nuc PC Growth Cones Turn Dorsally at the Hindbrain Intersection in the Absence of the TPOC}

In order to assess the role of the TPOC axons for pathfinding by the nuc PC growth cones through the anterior hindbrain intersection, the TPOC axons, which are projected by neurons in the forebrain, were surgically prevented from extending into the midbrain. Previously we found that this manipulation induced a significant proportion of nuc PC growth cones to follow aberrant pathways in the anterior tegmental intersection, but $61 \%$ of the growth cones still followed an apparently normal longitudinal pathway through the dorsolateral tegmentum (Chitnis and Kuwada, 1991). These latter growth cones enter the anterior hindbrain intersection, but were much more likely to turn dorsally than would the control growth cones (Fig. 6, 7). A comparison of the angles made by nuc PC growth cones with the plane perpendicular to the longitudinal axis both in the presence and absence of the TPOC showed that growth cones significantly turned dorsally more often in the absence of the TPOC (Wilcoxon rank sum test: control $n$
$=35$, experimental $n=18, T=351, p<0.01)$. Furthermore, the growth cones that turned dorsally extended farther in the dorsal direction in the absence of the TPOC than in the presence of the TPOC (Wilcoxon rank sum test: control $n=9$, experimental $n=13, T=74, p<0.025$ ). Corroborating these findings were four nuc $\mathrm{PC}$ axons that looped at the anterior hindbrain intersection following the elimination of the TPOC (Fig. 8). Such loops were never seen in unmanipulated embryos. Similar loops were also seen in nuc PC axons at the intersection in the anterior tegmentum following elimination of the TPOC (Chitnis and Kuwada, 1991).

\section{DISCUSSION}

\section{Growth Cone Guidance at the Anterior Tegmental Intersection}

The behavior of nuc PC growth cones at the intersection in the anterior tegmentum following the elimination of the TPOC suggested that (1) selective fasciculation with TPOC axons by nuc PC growth cones normally participates in guiding these growth cones and (2) cues in the dorsolateral tegmentum independent of the TPOC axons may also help guide these growth cones (Chitnis and Kuwada, 1991). Our analysis of pathfinding through this intersection corroborates and extends this hypothesis. First, our ultrastructural examination of nuc PC growth cones confirms that they normally fasciculate with the PC axons then with TPOC axons. This is consistent with a role for se- 

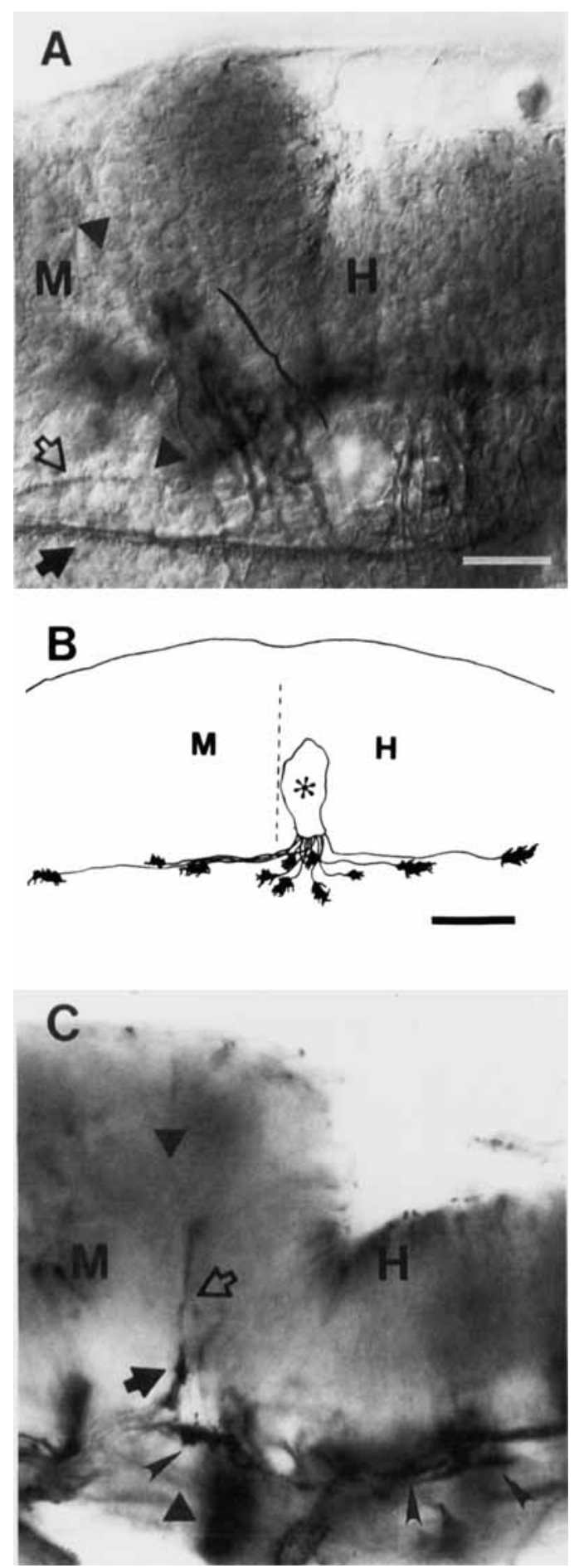

Figure 5 nuc PC growth cones encounter an intersection of tracts in the anterior hindbrain. (A) Acetylated $\alpha$-tubulin labeling of axons reveals axons of anterior hindbrain neurons (bracket) that extend ventrally to intersect the TPOC (open arrow) and MLF (closed arrow) axons at $22 \mathrm{~h} \mathrm{PF}$. The border between the midbrain (M) and hindbrain $(\mathrm{H})$ is denoted by triangles. The labeled cells, which are out of the focal plane of this micrograph and partially obscure the hindbrain neurons, are trigemi- lective fasciculation. Additionally, nuc $\mathrm{PC}$ growth cones that follow their normal pathway despite the absence of the TPOC do so by extending on what are likely the surfaces of neuroepithelial cells found in the dorsolateral tegmentum with little contact with the basal lamina. Consequently neuroepithelial cells in the dorsolateral tegmentum are a potential source of guidance cues. The endfeet of neuroepithelial cells likely provide guidance cues to growth cones in a variety of organisms since growth cones in the amphibian spinal cord (Nordlander and Singer, 1978; Singer, Nordlander, and Egar, 1979; Nordlander and Singer, 1982), chick optic stalk (Silver and Rutishauser, 1984) and spinal cord (Holley, 1987; Yaginuma, Homma, Kunzi, and Oppenheim, 1991), mouse optic nerve (Silver, 1984), zebrafish brain (Wilson and Easter, 1991), and the Japanese medaka fish spinal cord (Kuwada, 1986) also extend in contact with neuroepithelial endfeet. In the chick optic stalk the neuronal cell adhesion molecule, N-CAM, found on neuroepithelial endfeet may be involved in guiding optic growth cones (Silver and Rutishauser, 1984). If the endfeet in the dorsolateral tegmentum do provide guidance cues to the nuc PC growth cones, then it is possible that the same cues that guide the pioneer TPOC growth cones through the tegmentum may also be capable of guiding follower nuc PC growth cones. Such a proposition may also apply to other embryos, for example, elimination of pioneer neurons in the grasshopper limb did not prevent one set of follower growth cones from following their normal pathway (Keshishian and Bentley, 1983).

Second, in our fixed embryos, nuc PC growth cones appeared to turn onto the TPOC axons without making extensive contact with the MLF. This

nal ganglion cells that are located under the epidermis in this region. (B) Camera lucida drawing of the dil-labeled growth cones of the anterior hindbrain cells at $26 \mathrm{~h}$ PF. Asterisk denotes the site of dil injection; dashed line denotes the midbrain/hindbrain border. (C) Micrograph showing a photooxidized, dil-labeled nuc PC growth cone (closed arrow) turning dorsally onto an acetylated $\alpha$-tubulin-labeled hindbrain axon (open arrow) in a 27h-old embryo. The other dil-labeled nuc PC growth cones (closed arrowheads) have extended on the TPOC axons beyond the anterior hindbrain intersection without turning. Out of the focal plane are trigeminal ganglion cells that partially obscure the growth cones in the hindbrain. A midbrain neuron that was backlabeled with dil can be also seen out of the focal plane. Scale bar $=25$ $\mu \mathrm{m}(\mathrm{A}, \mathrm{C})$; and $50 \mu \mathrm{m}$ (B). 
A

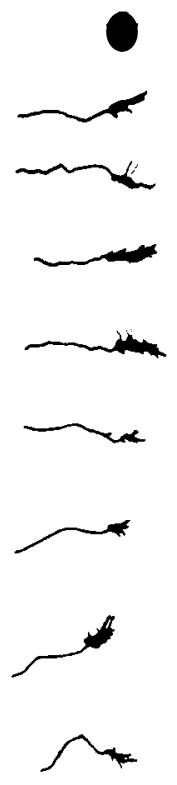

*
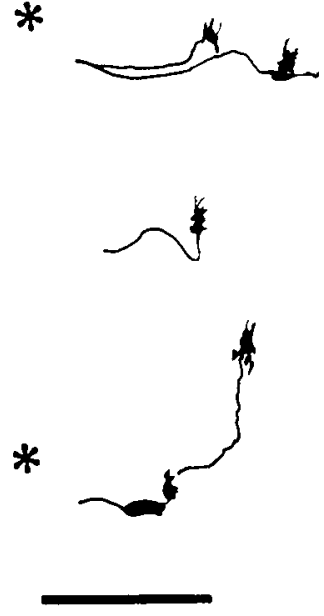

B
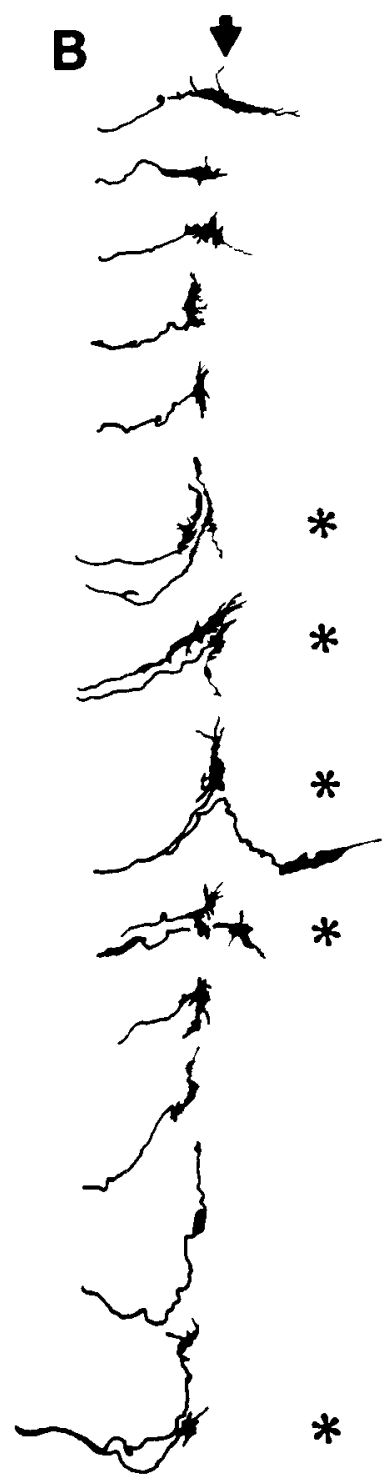

*

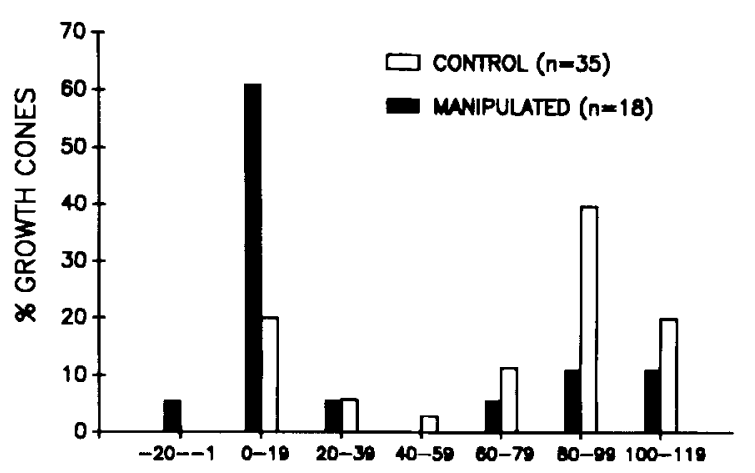

ANGLE MADE BY GROWTH CONES WITH VERTICAL PLANE

Figure 7 The distribution of the orientations of nuc PC growth cones at the anterior hindbrain intersection. The abscissa represents the angle made by the nuc PC growth cones with the plane of the midbrain/hindbrain boundary. Dorsally oriented growth cones were clustered around $0^{\circ}$, whereas longitudinally oriented growth cones clustered around $90^{\circ}$. The growth cones oriented between $-1^{\circ}$ and $-20^{\circ}$ had turned dorsally and slightly anteriorly. The ordinate represents the frequency with which growth cones from 30 control and 13 manipulated embryos were found at any particular orientation.

tend on the MLF in the absence of the TPOC, it seems unlikely that active inhibition plays a major role in preventing the growth cones from extending on the MLF.

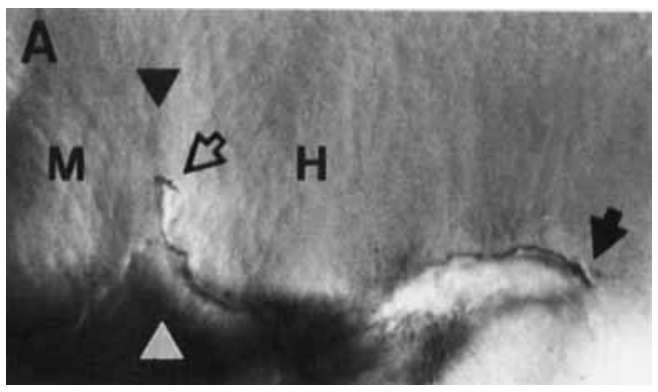

B

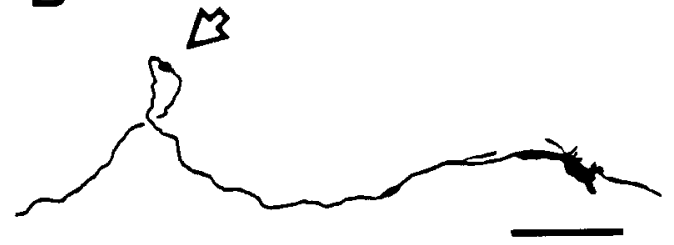

Figure 8 A micrograph (A) and camera lucida drawing (B) of a dil-labeled nuc PC axon that looped (open arrow) at the anterior hindbrain intersection following the elimination of the TPOC. The growth cone (closed arrow) of this axon had extended into the hindbrain $(\mathrm{H})$. Triangles denote the midbrain $(\mathrm{M}) /$ hindbrain border. In the lower left portion of the panel are melanocytes that are out of the focal plane. Scale bar $=25 \mu \mathrm{m}$. 
Third, each nuc PC growth cone may be capable of independently choosing an appropriate pathway at the tegmental intersection. Previously it was demonstrated that in the absence of the TPOC, nuc PC growth cones often followed different pathways in the same embryo (Chitnis and Kuwada, 1991). Concordantly, we found that occasionally two nuc $\mathrm{PC}$ growth cones that had reached the intersection at approximately the same time turned onto separate TPOC bundles. Both findings suggest that nuc $\mathrm{PC}$ growth cones need not obligatorially follow each other.

Fourth, cues that guide the nuc PC growth cones must provide two kinds of information: (1) information that allows nuc PC growth cones to select the appropriate pathway; and (2) information that allows nuc PC growth cones to extend in the correct direction along the selected pathway. Our studies have shown that cues associated with the TPOC and perhaps with the endfeet of dorsal neuroepithelial cells help nuc PC growth cones to select their normal dorsolateral path in the tegmentum. However, since nearly all nuc PC growth cones extend caudally in the absence of the TPOC, adequate directional cues independent of the TPOC are likely to be available in the tegmental intersection.

\section{Growth Cone Guidance at the Anterior Hindbrain Intersection}

The analysis of nuc $P C$ growth cones in the absence of the TPOC suggests that TPOC axons help guide them at the anterior hindbrain as well as in the anterior tegmental intersection. First, following the elimination of the TPOC, growth cones increased the frequency with which they turned dorsally onto the anterior hindbrain axons. The growth cones that did turn aberrantly extended dorsally for a longer distance than did the occasional growth cone that turned dorsally in normal embryos. Second, occasionally, nuc PCaxons looped in the anterior hindbrain intersection in the absence of the TPOC. The loop likely resulted when nuc PC growth cones extended dorsally for some distance but then corrected their trajectory to extend caudally. Similar loops were observed in the anterior tegmental intersection following the elimination of the TPOC (Chitnis and Kuwada, 1991). Axon loops and hairpin turns that presumably represent similar course corrections have also been observed following experimental manipulation in several other embryos, for example, sensory and motor axons in chicks (Tosney, 1988) and Mauthner axons in amphibian embryos (Stefanelli, 1951).
Our results suggest that the anterior hindbrain intersection represents a second region of uncertainty for nuc PC growth cones and that fasciculation with the TPOC axons normally helps the growth cones pass through this region without error. However, as in the anterior tegmental intersection, the role played by the TPOC axons is not obligatory: a significant proportion of nuc PC growth cones extends through the hindbrain intersection without error despite the absence of the TPOC axons. This suggests that multiple sources of guidance cues may normally insure that nuc PC growth cones follow their correct pathway through this intersection. Similarly, multiple guidance cues may also direct nuc PC growth cones at the anterior tegmental intersection (Chitnis and Kuwada, 1991) and spinal growth cones near the ventral midline in the spinal cord (Bernhardt, Nguyen, and Kuwada, 1992) in the zebrafish embryo.

Multiple, redundant guidance cues for growth cones are likely to be employed by a variety of other embryos. Redundant adhesion systems have been directly demonstrated for chick growth cones in vitro by both antibody block experiments (Tomaselli, Neugebauer, Bixby, Lilien, and Reichardt, 1988 ) and genetic manipulation of putative pathfinding molecules in Drosophila embryos (Elkins, Zinn, McAllister, Hoffman, and Goodman, 1990). Furthermore, elimination of putative guidance cues leads growth cones to follow both normal and aberrant pathways in Drosophila (Schubiger and Palka, 1985), grasshoppers (Raper, Bastiani, and Goodman, 1984; Harrelson and Goodman, 1988), and nematodes (Hedgecock, Culotti, and Hall, 1990) as it does in the zebrafish. Further analyses of growth cone guidance, especially in simple, manipulative embryos, such as those of zebrafish, should be useful in identifying multiple sources of guidance cues.

We thank G. Piperno for the monoclonal antibody against acetylated $\alpha$-tubulin, P. Knafl for technical assistance, D. Bay for photography, and B. Oakley for critical reading of this manuscript. This research was supported by a grant from NIH (NS24848). A.B.C. was supported by a Rackham Fellowship at the University of Michigan and C.K.P. was supported by a NSF grant (DIR9014275 ) to the Research Training Group in Developmental Neurobiology at the University of Michigan.

\section{REFERENCES}

Bernhardt, R. R., NGuyen, N., and Kuwada, J. Y. (1992). Growth cone guidance by floor plate cells in 
the spinal cord of zebrafish embryos, Neuron 8:869882

Chitnis, A. B. and Kuwada, J. Y. (1990). Axonogenesis in the brain of zebrafish embryos. J. Neurosci. 10:1892-1905.

Chitnis, A. B. and Kuwada, J. Y. (1991). Elimination of a brain tract increases errors in pathfinding by follower growth cones in the zebrafish embryo. Neuron 7:277-285.

DODD, J. and Jessell, T. M. (1988). Axon guidance and the patterning of neuronal projections in vertebrates. Science 242:692-699.

ElKins, T., ZINN, K., MCAllister, L., HofFMAN, F. M., and GoOdMan, C. S. (1990). Genetic analysis of a Drosophila neural cell adhesion molecule: interaction of fasciclin I and Abelson tyrosine kinase mutations. Cell 60:565-575.

Goodman, C. S., Bastiani, M. J., Doe, C. Q., DU LaC, S., Helfand, S. L., Kuwada, J. Y., and Thomas, J. B. (1984). Neuronal recognition during development: cellular and molecular approaches. Science 225:1271-1279.

Harrelson, A. L. and GoOdman, C. S. (1988). Growth cone guidance in insects: fasciclin II is a member of the immunoglobulin superfamily. Science 242:700-708.

Hedgecock, E. M., Culotti, J. G., and Hall, D. H. (1990). The unc-5, unc-6, and unc-40 genes guide circumferential migrations of pioneer axons and mesodermal cells on the epidermis in C. elegans. Neuron 4:61-85.

Holley, J. A. (1987). Differential adhesivity of neuroepithelial cells and pioneering circumferential axons. Dev. Biol. 123:389-400.

HoNig, M. G. and HuME, R. I. (1986). Fluorescent carbocyanine dyes allow living neurons of identified origin to be studied in long-term cultures. J. Cell Biol. 103: $171-187$.

Keshishian, H. and BentLey, D. (1983). Embryogenesis of peripheral nerve pathways in grasshopper legs III. Development without pioneer nerves. Dev. Biol. 96:116-124.

KIRCHEN, R. V. and WEST, W. R. ( 1976). The Japanese Medaka, its care and development. Carolina Biological Supply Co., Burlington, NC.

KuwadA, J. Y. (1986). Cell recognition by neuronal growth cones in a simple vertebrate embryo. Science 233:740-746.

KuWADA, J. Y., Bernhardt, R. R., and ChITNIS, A. B. (1990). Pathfinding by identified growth cones in the spinal cord of zebrafish embryos. J. Neurosci. 10:1299-1308.

LANDMESSER, L. (1986). Axonal guidance and the formation of neuronal circuits. Trends Neurosci. 9:489492.

Maranto, A. R. (1982). Neuronal mapping: a photooxidation reaction makes Lucifer yellow useful for electron microscopy. Science 217:953-955.

MYers, P. Z., EISEN, J. S., and Westerfield, M. (1986). Development and axonal outgrowth of identi- fied motoneurons in the zebrafish. J. Neurosici. 6:2278-2289.

Nordlander, R. H. and Singer, M. (1978). Role of ependyma in regeneration of the spinal cord in the urodele amphibian tail. J. Comp. Neurol. 180:349374.

Nordlander, R. H. and Singer, M. (1982). Morphology and position of growth cones in the developing Xenopus spinal cord. Dev. Brain Res. 4:181-193.

Piperno, G. and Fuller, M. T. (1985). Monoclonal antibodies specific for an acetylated form of $\alpha$-tubulin recognize the antigen in cilia and flagella from a variety of organisms. J. Cell Biol. 101:2085-2094.

Raper, J. A., Bastiani, M. J., and Goodman, C. S. (1984). Pathfinding by neuronal growth cones in grasshopper embryos. IV. The effects of ablating the A and $P$ axons upon behavior of the $G$ growth cone. $J$. Neurosci. 4:2329-2345.

SChubiger, M. and Palka, J. ( 1985 ). Genetic suppression of putative guidepost cells: effect on establishment of nerve pathways in Drosophila wings. Dev. Biol. 108:399-410.

Silver, J. (1984). Studies on the factors that govern directionality of axonal growth in the embryonic optic nerve and the chiasm of mice. J. Comp. Neurol. 223:238-251.

Silver, J. and Rutishauser, U. (1984). Guidance of optic axons in vivo by a preformed adhesive pathway on neuroepithelial endfeet. Dev. Biol. 106:485-499.

Singer, M., Nordlander, R. H., and EGAR, M. (1979). Axonal guidance during embryogenesis and regeneration in the spinal cord of the newt: the blueprint hypothesis of neuronal pathway patterning. $J$. Comp. Neurol. 185:1-22.

Stefanelli, A. (1951). The Mautherian apparatus in the icthyopsida; its nature and function and correlated problems of neurohistogenesis. Quaterly Rev. Biol. 26:17-34.

Tomaselli, K. J., Neugebauer, K. M., Bixby, J. L., LILIEN, J., and REICHARDT, L. F. ( 1988). N-Cadherin and integrins: two receptor systems that mediate neuronal process outgrowth on astrocyte surfaces. Neuron 1:33-43.

TOSNEY, K. W. (1988). Proximal tissues and patterned neurite outgrowth at the lumbosacral level of the chick embryo: partial and complete deletion of the somite. Dev. Biol. 127:266-286.

Wilson, S. W., Ross, L. S., Parrett, T., and Easter, S. J. (1990). The development of a simple scaffold of axon tracts in the brain of the embryonic zebrafish, Brachydanio rerio. Development 108:121-145.

Wilson, S. W. and Easter, S. J. (1991). A pioneering growth cone in the embryonic zebrafish brain. Proc. Natl. Acad. Sci. USA 88:2293-2296.

Yaginuma, H., Homma, S., Kunzi, R., and OPPENHEIM, R. W. (1991). Pathfinding by growth cones of commissural interneurons in the chick embryo spinal cord: a light and electron microscopic study. J. Comp. Neurol. 304:78-102. 